Notre Dame Journal of Formal Logic

Volume V, Number 2, April 1964

\title{
REMARKS ON THE COMPLETENESS OF LOGICAL SYSTEMS \\ RELATIVE TO THE VALIDITY-CONCEPTS \\ OF P. LORENZEN AND K. LORENZ. ${ }^{1}$
}

\author{
WOLFGANG STEGMÜLLER
}

1. The so-called semantics of elementary logic (predicate logic of first order) has some peculiarities which may be considered as disadvantages, at least if looked at from a certain point of view. First of all, it makes use of a very strong set-theoretical apparatus which is highly non-constructive. Strict constructivism may not be obtainable for a semantic foundation of logic; but even if one does not subscribe to constructivism one should expect that a weaker apparatus would be sufficient to define logical validity or logical implication on the elementary level. Secondly this set-theoretical approach is restricted to classical logic and can therefore not be used, e.g. to define a concept of validity for intuitionistic logic or other logical systems differing from the classical one. This fact will not be considered as a drawback by those for whom classical logic is the only "real" logic. On the other hand "classicists" as well as "intuitionists" should welcome an account of logic with the help of which different logical systems can be compared on the basis of semantical concepts alone-an account in which the deviations of logical systems from each other would be mirrored by different concepts of validity. Thirdly there are two characteristics of this semantics based upon the Bolzano-Tarski approach which in various contexts have been the main points of attack in the arguments of intuitionists and constructivists against this approach (which are mistakenly thought to be arguments againist classical logic): (a) the procedure of introducing logical connectives as truth-functions tacitly presupposes that every meaningful sentence is either true or false (true-false-alternative). In view of such urproved and unrefuted sentences as "there is at least one odd perfect number" one can reasonably doubt whether this assumption is correct.

1. This paper was originally intended to be part of a philosophical article from which it had to be separated because of its technical character. The philosophical remarks in the introductory section are not elaborated and serve only the purpose of facilitating a better understanding of the following parts (by contrast and comparison). The paper is selfcontained. No previous knowledge of the works of P. Lorenzen and K. Lorenz is presupposed. 
Namely if one decides to identify in mathematical contexts "true" with "provable" and "false" with "refutable" it is certainly not justified. And if one does not accept this identification then if one doesn't want to destroy the meaning of "true" altogether it seems hardly possible to do without some kind of "ontological hypothesis" about the pre-existence of numbers or other kinds of mathematical entities; but the best that can be said about such a hypothesis is that it is in need of a further justification. Contrary to a widespread belief the meaningfulness of sentences does not always rest upon the true-false-alternative. With respect to the given example the thesis of its meaningfulness can be based on the fact that-using an innocuous counter-factual-it is known what would be accepted as a proof of it. (b) The definition of validity contains a universal quantifier ranging over all nonempty universes. The inclusion of infinite universes is essential. A person to whom the concept of a closed infinite totality does not make sense would interpret the quantifier "for all non-empty universes" in the definition of validity as "for all non-empty finite universes" and thereby get a different logic. In this indirect way the concept of a closed infinite set enters into the definition of logical validity. One could say that all the philosophers of the past who rejected the possibility of actual infinity could either not have accepted this concept of validity or would necessarily have misinterpreted it in the way just indicated.

2. P. Lorenzen's recent account of logical connectives and quantifiers given in [2] and [3] can best be interpreted as a new kind of semantics as has been done in [1] by K. Lorenz. This semantics differs in some essential respects from the Bolzano-Tarski approach: First, the two objections (a) add (b) can not be made any more because it is free from the true-falsealternative and the concept of infinite set does not enter into the definition of validity. Secondly, it turned out to be more flexible than the set-theoretical approach as this method makes it possible to define validity for different logical systems, especially for classical and intuitionistic logic (though this feature apparently is not quite in accordance with Lorenzen's original intention). As far as the important question of constructivity is concerned this point will shortly be discussed later. Anticipating the result of this discussion we can say that it is not constructive in the strict sense. But allowing the use of a comparative instead of a classificatory concept of constructivity, it seems to be more constructive and the technical apparatus needed seems to be weaker than concept formations used within the settheoretical approach.

Technically speaking Lorenzen's approach differs from the usual one by the use of concepts which belong to the theory of games instead of concepts that belong to set theory. We therefore distinguish this type of semantics-the game-theoretical semantics-from the set-theoretical semantics of the Bolzano-Tarski approach. A few remarks about the term "semantics" seem to be in order here. First this semantics does not "abstract from" the users of the language. An account for which this holds true is sometimes called "pragmatics" rather than "semantics." If the motivation for this distinction is based on the assumption that every explicit 
reference to the users of a language must make the investigation in question an empirical one (R. Carnap, "Introduction to Semantics," $\$ 4$ and 5, especially $\left.p_{\circ} 13\right)$, it would be wrong in the present case. This simply follows from the fact that the reference made does not differ from the reference to the players in the mathematical theory of games, which is obviously not an empirical science. Secondly the occurrence of terms like "designates," "names," "denotes," "refers to" etc. among the primitives is sometimes considered as a criterion for what properly should be called "semantics;" but the game-theoretical semantics does not contain such terms. Here it may be sufficient to notice that all the terms quoted are applicable only to descriptive signs. What is at stake within formal logic is the meaningassignment to logical signs so that we can abstract from the question what the meanings of descriptive terms consist in and what these terms refer to. But of course in case there should be other and more cogent reasons for not using the name "semantics" to the game-theoretical interpretation of logical signs and the theory erected upon this interpretation no objections should be made against exchanging this word for a better one. Perhaps one should not take too serious the question of what can be properly called 'semantics'. The use of this term, which was originally intended to designate the theory of meanings (read: intensions) for a purely referential theory like the Bolzano-Tarski approach to logic is highly artificial anyway. The expression "mengentheoretische Pradikatenlogik" ("set-theoretical predicate logic") as used in Hilbert-Bernays, "Grundlagen der Mathematik", was a better term insofar as it clearly says what kind of tools are used.

3. The main part of this paper will consist of a detailed completeness proof of intuitionistic logic relative to the game-theoretical definition of validity. This logic was chosen for the proof because it is the strongest and presumably the most interesting among those logical systems for which no intuitively satisfactory concept of validity had previously been defined. At the end some hints will be given as to how this method can be used to prove the completeness of three other logical systems including the classical one. In many details we will avail ourselves of explications and technical improvements of Lorenzen's original characterization of the new approach which are due to K. Lorenz (vid. [1]). We especially accept from the thesis of $\mathrm{K}$. Lorenz the following items: the way of characterizing the asymmetry between the two players considered, the concept of (open and closed) round, the explicit formulation of a rule which corresponds to what is here called "structural rule," the concept of the reduced tableau and the gentzen-like calculus (with minor modifications). What is new in this proof is the systematic use of tree-constructions. Apart from the fact that this method makes a quasi-constructive proof of the completeness and soundness of certain calculi possible it will have some further advantages: it generally facilitates the application of game-theory to logic; it makes easier the comparison with other investigations of intuitionistic logic in which trees play an essential role; it finally establishes a link between fundamental concepts of logic and of Brower's set theory ("finitary spread," "choice sequence"). The branches of the trees to be considered will represent choice sequences 
in the most literal sense of the word. The construction used will therefore help to answer the question about the degree of constructivity of the concepts involved.

4. For the sake of illustration the type of games to be considered will be characterized by using some elementary set-theoretical terms: We have two players, called $W$ (White) and $B$ (Black), a set $M$ of positions (each of which in the logical application will be represented by a tableau) and a rule $R$ which formally is a two-placed relation obtaining between position. If $x R y$ for positions $x$ and $y$ we say that $x$ has been transformed into $y$ by a permissible move. $M$ is subdivided into two exclusive domains: the domain (of moves of) $W$ and the domain of $B$, called $M_{W}$ and $M_{B}$. Using the terminology of $\mathrm{K}$. Lorenz we further require that $R$ be compatible with this subdivision in the sense that a pair $x ; y$ belongs to $R$ iff $x$ and $y$ respectively belong to different subdomains (intuitively speaking no player is allowed to make two moves in succession). $R$ will usually be a many-many relation. If $x R y$ then $x$ is called an $R$-predecessor of $y$ and $y$ an $R$-successor of $x$. If to a given $x$ there is no $z$ such that $x R z$ (i.e. no $R$-successor of $x$ is defined) then $x$ is called an end-position.

We distinguish between a game as a type and a "concrete performance" of a game or a tournament. In a tournament either $B$ or $W$ has to produce the initial position of the tournament in the zero-step. Because of the asymmetry between $B$ and $W$ introduced below the class of tournaments of a type of game thereby splits up into two proper sub-classes. If an initial position $x$ after a finite number of moves (each made in accordance with $R$ ) is transformed into an end-position $e$ which is an element of $M_{B}^{\prime}$ then $W$ has won the tournament (because this means that the next move would be up to $B$ but $B$ is not allowed to make a move by the rule of the game). $W$ wins only in this case. If $e$ belongs to the domain of $W$ then $B$ has won. There is no necessity that an end-position must be reached in a finite number of steps. Such a tournament formally of infinite length could be considered as a typical candidate for a draw. But no draw shall be admitted in the games considered. Rather in such a situation it is stipulated that $B$ has won. This introduces the first asymmetry between the two players: $B$ not only wins by reaching an end-position favourable to him in a finite number of steps but as well by preventing $W$ from reaching such an end-position favourable to $W$.

5. Now we come to the application to logic. The following logical signs are used: $\wedge, \vee, \neg, \rightarrow, \wedge, \vee$. Within the metalanguage we use Greek capitals to designate formulas, small Greek letters to designate individual variables, " $a$ " (without or with subscript) to designate individual constants (i.c.) taken from a potentially infinite list of such constants and " $p$ " to designate prime formulas. The latter are either propositional letters or predicate letters with an appropriate number of individual variables or constants attached. Only closed formulas (i.e. formulas without free individual variables) will be considered.

Lorenzen's basic idea can be described like this: The meanings of logical signs are not determined with the help of truth-conditions but by a precise description of their function in language-games of a special sort, 
called dialogue-games; more exactly: the meaning of a logical sign is determined by specifyng how sentences (formulas) containing it as the principal logical sign after having been presented by one player (the "proponent") may be attacked by the counter-player (the "opponent") and defended against this attack by the first player.

The dialogue-games are special kinds of games falling under the abstract schema described above. The names "proponent" $(P)$ and "opponent" $(O)$ shall be used not to designate special players but only to characterize a momentary situation in the game with respect to any of the two players (this use is different from Lorenzen's who usually calls that player "proponent" whom we name "global validity-proponent" and "opponent" the counter-player of this one): whoever attacks a move of his counterplayer is in this function opponent and whoever presents either the very first formula or another formula in a move attacked later is in this function proponent. $^{2}$ The player who in the zero-step sets forth a formula is called the global proponent and his counter-player the global opponent. If the global proponent is identical with $W$ we call him the (global) validity-proponent because he will represent the validity-claim with respect to the formula having been set forth in the zero-step; $B$ is in this case called the (global) validity-opponent. On the other hand $B$ in the role of the global proponent represents the satisfiability-claim and is to be called the (global) satisfiability-proponent and $W$ in this situation the (global) satisfiability-opponent.

The moves of the game are of two kinds: 1) setting forth closed formulas and 2) making challenges: ? (dubito), $\stackrel{?}{\ell}$ (dubito left), $\stackrel{?}{r}$ (dubito right), $\stackrel{?}{a}$ (dubito for a) (a is an i.c.).

The rule $R$ consists of three parts: the logical rule $L$, the basic rule $B$ and the structural rule $S$.

$L$ is so-to-speak the core of the game-rule. Suppose the proponent has set forth the formula $\Phi$. $L$ prescribes which attacks are permissible and what defence moves are possible against these attacks: 1) if $\Phi$ is $\Phi_{1} \wedge \Phi_{2}$ then the opponent can choose between the two moves ${ }_{l}$ and ${ }_{r}$. In the first case the only possible defence consists in setting forth $\Phi_{1}$ and in the second case in setting forth $\left.\Phi_{2} ; 2\right)$ if $\Phi$ is $\Phi_{1} \vee \Phi_{2}$ then the only possible attack is ?; $P$ can defend by his own free choice by setting forth $\Phi_{1}$ or $\left.\Phi_{2} ; 3\right)$ if $\Phi$ is $\neg \Phi_{1}$ then the only possible attack consists in setting forth $\Phi_{1}$. No defence is possible (i.e. $P$ can make only other kinds of counter-moves if they are permitted by the rules, e.g. to attack $\Phi_{1}$ ); 4) if $\Phi$ is $\Phi_{1} \rightarrow \Phi_{2}$ then the only possible attack consists in setting forth $\Phi_{1}$ and the only possible defencemove of $P$ consists in setting forth $\Phi_{2}$ (but $P$ can make other moves if they are allowed by the rules; these rules allow in all cases to choose an attack against the $\Phi_{1}$ of $O$ instead of defending against his attack); 5) if $\Phi$ is $\wedge \alpha \Phi_{1}$ then $O$ can choose between infinitely many possible attacks: the attack move consists in ? whereby the i.c. $a$ is picked out by 0 according to his own free

2. So a player can be opponent and proponent even with respect to one and the same move, depending on whether we relate this move to a previous or to a later move of his counter-player. 
choice. The only possible defence reaction of $P$ is $\left.\Phi_{1}\left(\begin{array}{l}\alpha \\ a\end{array}\right)^{3} ; 6\right)$ if $\Phi$ is $\vee \alpha \Phi_{1}$ then the attack consists in the move ?. The defence is $\Phi_{1}\left(\begin{array}{l}\alpha \\ a\end{array}\right)$ whereby this time $P$ can arbitrarily choose a from the potentially infinite list of i.c.

The basic rule $\mathbf{B}$ imposes upon $W$ restrictions in the use of (closed) prime formulas within attack- or defence-moves: $W$ is allowed to use a prime formula in such a move only if the same prime formula has been set forth in a previous move by $B$. There is no restriction in the use of prime formulas for $B$. It goes without saying that prime formulas may never be attacked within a dialogue.

Before formulating the last part of the game-rule it is necessary to characterize the formal representation of dialogues and positions. Taking a word from Beth this representation is made with the help of tableaux. By a tableau we understand a diagram in which the expressions designating moves are inserted. It consists of a $B$-column on the left and a $W$-column on the right. The dialogue is subdivided into rounds each represented by one line of the tableau. The rounds are counted (without explicit numeration) starting on top of the diagram, the upper-most round being called the zero-round. The first move presenting the initial formula of the dialogue opens the zero round which will never be closed. In the following steps an attack always opens a new round (following the last round which contains a symbol for a move) and a defence against the attack closes the same round. The latter can happen only if a defence is possible (what is not the case if the attack was made against a $\urcorner$-formula). We sometimes say that an attack is made against a formula or against a round meaning that it is made against a move consisting of setting forth this formula or a move in that round. As attack moves can be made not only against formulas in the immediately preceding round but against formulas in earlier rounds as well, the attack move is to be accompanied by a number indicating the round against which the attack was made. This numeration has the further purpose of telling us for each closed round occurring in a tableau which of the two moves in this line is the attack (with number attached) and which is the defence (without number). If one wants to use a single tableau to read off the whole course of a dialogue then in addition to the first use of numbers a second numeration indicating the steps is to be used (as in the general case a player is allowed to "jump back" to close an earlier round). But this numeration is not necessary. From a formal point of view one can identify a position in a concrete dialogue $D$ reached after move $m$ has been made with that part of the tableau depicting $D$ which was obtained in the moment the symbol destgnating $m$ had been inserted.

The structural rule $\mathbf{S}$ specifies the circumstances and the number of times attacks and defences can be made (the logical rule doesn't say anything about this): 1) $B$ may attack once (and only once) by means of an attack move in a round $k$ a formula of the $W$-column occuring in a round $i$ if $k>i$; 2) $W$ may attack an arbitrary number of times a formula of the

3. This is the result of substituting a for the free occurrences of $\alpha$ in $\Phi_{1}$. 
$B$-column occuring in a round $i$ in case the attack is made in a round $k$ with $k>i$; 3) $B$ as well as $W$ may defend a formula against an attack made in round $i$ by a defence move made in the same round (thereby closing round $i$ ) if all rounds $k>i$ containing moves are closed(this part of the rule requires especially that for the closure of open rounds a certain order has to be observed so that later open rounds have to be closed before the earlier ones (if at all); a further consequence of this part of the rule will be discussed later).

We can now define: The rule $\mathbf{R}$ of the game is the logical rule $\mathbf{L}$ restricted and regulated in application by the basic rule and the structural rule. The name "structural rule" was used for the third part of $\mathbf{R}$ because it determines the structure of the dialogue-game as intuitionistic, classical etc. If it is formulated in the way described above then it turns out that we get the intuitionistic logic.

( $L$ and $B$ have been explicitly formulated by P. Lorenzen; $S$ constitutes what $\mathrm{K}$. Lorenz called "special rule", $\mathrm{L}$ is his "general rule" and B what is called the supplement to the general rule).

Win and loss are defined as in the abstract case: $W$ has won a dialogue iff after a finite number of moves a position was reached (formally represented by a tableau $X$ ) which is in the domain of $B$ but for which no $R$-successor is defined ( $B$ can make no further move). In all the other cases $B$ wins.

A player $p_{i}$ has chosen a strategy $S$ with respect to an initial formula $\Phi$ if for each possible move of his counter-player (i.e. for each corresponding tableau in the domain of $p_{i}$ ) the $R$-successor is uniquely determined in advance, the zero-move consisting of setting forth the formula $\Phi . p_{i}$ can either be identical with the player who has set forth this initial formula or can be different from him. Formally speaking such a strategy is a function of higher order which for every $X$ in the domain of $p_{i}$ uniquely determines an $R$-successor whereby the first $X$ contains the formula in question only. The concept of a strategy could be introduced in a completely general way (i.e. without reference to a special formula) but for the applications needed the relativised concept will do.

We sometimes use the symbol " $X(\Phi)$ " to designate a tableau whose initial formula is $\Phi$ (this symbol is ambiguous as it does not tell whether the formula appears on the right or on the left upper corner of the tableau, i.e. whether it has been set forth by $W$ or by $B$ in the zero-move). Similarly we use " $D(\Phi)$ " to designate a dialogue starting from this formula. A strategy $S$ (with respect to the initial formula $\Phi$ ), chosen by player $W$, is called a $W$-win-strategy for the initial position (which may be either in the domain of $B$ or in that of $W$ ) iff $W$ by making his moves in accordance with $S$ wins every dialogue $D(\Phi)$ beginning with this initial position, i.e. he reaches in a finite number of moves an end position $X(\Phi)$ favourable for him (in the domain of $B$ ), no matter what moves $B$ chooses for the tableaux $X(\Phi)$ in $M_{B}$. Similarly a strategy $S$ (with respect to an initial formula) chosen by player $B$ is a $B$-win-strategy for the initial position iff $B$ wins every dialogue starting from this initial position in case he makes all his moves as 
prescribed by $S$. It should be kept in mind that in these definitions of winstrategies the asymmetry between $B$ and $W$ comes into play: that $B$ wins every dialogue by choosing $S$ does not mean that he reaches in every case an end position favourable for him in a finite number of steps (as it would be the case for $W$ in place of $B$ ) but that he succeeds by reacting according to $S$ in preventing $W$ from winning. In this case only some of the dialogues will end after a finite number of steps whereas the others will formally be of infinite length. The latter will be the normal case because if one single formula appears on the $B$-column that may be attacked by $W$ in accordance with $L$ and $B$ this attack may be repeated by him an arbitrary number of times according to part 2) of the structural rule.

Validity and invalidity can now be introduced thus:

$D f_{1} . \Phi$ is valid $={ }_{\text {Df }}$ there exists a $W$-win-strategy for the initial position produced by $W$ in setting forth $\Phi$.

$D f_{2}$. $\Phi$ is invalid $={ }_{\text {Df }}$ there exists a $W$-win-strategy for the initial position produced by $B$ in setting forth $\Phi$.

Both concepts are reduced to $W$-win-strategies: in the first case $W$ successfully defends his validity claim for the formula he has set forth in the zero-move; in the second case $W$ successfully refutes the satisfiability ćlaim of $B$ for the formula $B$ has set forth in the zero-move. The dual concepts of satisfiability and rejectability which we shall not need in what follows could be introduced by reading in $\mathrm{Df}_{2}$ and $\mathrm{Df}_{1}$ " $B$-win-strategy" instead of " $W$-win-strategy."

It is not immediately clear in what relation these concepts stand to their analoga within set-theoretical semantics of classical logic. It turns out that they are the intuitionistic counterparts of these concepts (if the structural rule is chosen in the way it has been formulated).

In each concrete application an assertion of the form " $\Phi$ is valid" or " $\Phi$ is invalid" has to be used in the effective sense: a $W$-win-strategy of the kind required can effectively be given. Formally a win-strategy too can be represented by a tableau, making use in an obvious way of Beth's construction of subtableaux: A diagram representing a $W$-win-strategy must take into account all possible moves of $B$ if permitted by the rules. The same procedure can be used to show that no $W$-win-strategy exists: here a fixed $B$-strategy is represented by a diagram taking into account all possible choices of $W$ and showing that in no case $W$ reaches an end position favourable for him.

Remark 5.1. Using the concept of the closure of a tableau a calculus based upon the game-rules alone could be introduced in an analogous way to that chosen by Beth for the classical case with the help of his concept of a closed semantic tableau.

The following examples are used for illustration: 


\begin{tabular}{cc|c}
\multicolumn{2}{c|}{$B$} & $W$ \\
$\neg(p \vee \neg p)$ & 0 & \urcorner $า(p \vee \neg p)$ \\
$?$ & 2 & \urcorner $p$ \\
$p$ & 3 & \\
& & $1 p \vee\urcorner p$ \\
$?$ & 5 & $p$
\end{tabular}

The numbers indicate the rounds attacked. All the rounds before the last (closed) round that are open could not have been closed because they all stem from an attack against a $\neg$-formula. It is essential for the win of $W$ that he can (in round 5) repeat an earlier attack (made in round 2) against the formula in round 1 of the $B$-column: after his first counter-attack made in round 2 he lost the part of the dialogue ending with the $B$-attack in round 4 because $B$ used a prime formula for this attack; it is exactly this prime formula which $W$ can use after his second attack against the formula in round 1 to defend the iormula needed for this attack. $B$ never has a choice between different moves. So the tableau can not only be used to represent a concrete dialogue ending with win for $W$. It can be taken as formally representing a win-strategy for $W$. For this use the tableau is to be interpreted as a schema covering infinitely many cases differing from each other with respect to the prime formula which here remains arbitrary (whereas within its use to depict a concrete dialogue $p$ designates a particular prime formula or atomic sentence).

This example at the same time illustrates the fact that the last move of $W$ leading to win for him must always consist in "taking over" a prime formula from the $B$-column in order to make a permissible defence-move or a permissible attack-move. As long as $W$ uses a complex formula in one of his moves it can be attacked by $B$ within the next move and if $W$ uses a challenge it can be answered by $B$ because for $B$ there is no restriction upon the use of formulas.

It is clear that no analogous $W$-win-strategy for the case $p \vee \neg p$ exists: here $W$ can defend against the challenge of $B$ only by setting forth $\neg p$ and has lost as soon as this formula is attacked by $B$. This is one of the few cases where $B$ reaches an endposition favourable for him in a finite number of steps.

\begin{tabular}{|c|c|c|}
\hline$B$ & & $W$ \\
\hline & & $\vee \alpha \neg \Phi(\alpha) \rightarrow \neg \wedge \alpha \Phi(\alpha)$ \\
\hline$\vee \alpha\urcorner \Phi(\alpha)$ & 0 & $\neg \wedge \alpha \Phi(\alpha)$ \\
\hline$\wedge \alpha \Phi(\alpha)$ & 1 & \\
\hline$\neg \Phi(a)$ & & $?$ \\
\hline$\Phi(a)$ & & 2 \\
\hline & & $\Phi(a)$ \\
\hline
\end{tabular}


In this example $\Phi$ is supposed to be a prime formula. Again the tableau can be used not only to represent a concrete dialogue with win for $W$ but a schema representing a $W$-win-strategy because for $B$ there is always only one possible type of move. It is true that this time $B$ can choose the i.c. in his defence move in round 3 in infinitely many different ways; $W$ will win in all cases if he only chooses the same i.c. (in round 4) for his attack against the universal formula in round 2. So the schema-interpretation of the tableau this time not only covers all possible prime formulas taken as $\Phi$ but all possible i.c.'s taken as the $a$ as well.

In the case of the present formula $W$ gets a win-strategy as well if he slightly changes the order of moves made by him: for his second move he can decide to make a counter-attack instead of defending against $B$ 's first attack. This case illustrates what it means to "jump back" and close an earlier open round (if the later rounds are closed). We therefore depict this alternative too, this time-following $\mathrm{K}$. Lorenz-using an additional numbering on the outer borders to designate the order of moves:

\begin{tabular}{lrl|ccc}
\multicolumn{4}{c}{$B$} & \multicolumn{4}{c}{$W$} \\
\hline 1. & $\vee \alpha \neg \Phi(\alpha)$ & 0 & & $\neg \wedge \alpha \Phi(\alpha)$ & 4. \\
3. & $\neg \Phi(a)$ & & 1 & $?$ & 2. \\
5. & $\wedge \alpha \Phi(\alpha)$ & 1 & & & \\
7. & $\Phi(a)$ & & 3 & $?$ & 6. \\
& & & 2 & $\Phi(a)$ & 8.
\end{tabular}

Of course most cases are not as simple as the two mentioned: As soon as there exist for $B$ different types of reactions they have all to be taken into account either by constructing a tableau with nested subtableaux or by constructing a set of separate tableaux.

We now give a simple negative example which at the same time illustrates the method of subtableaux:

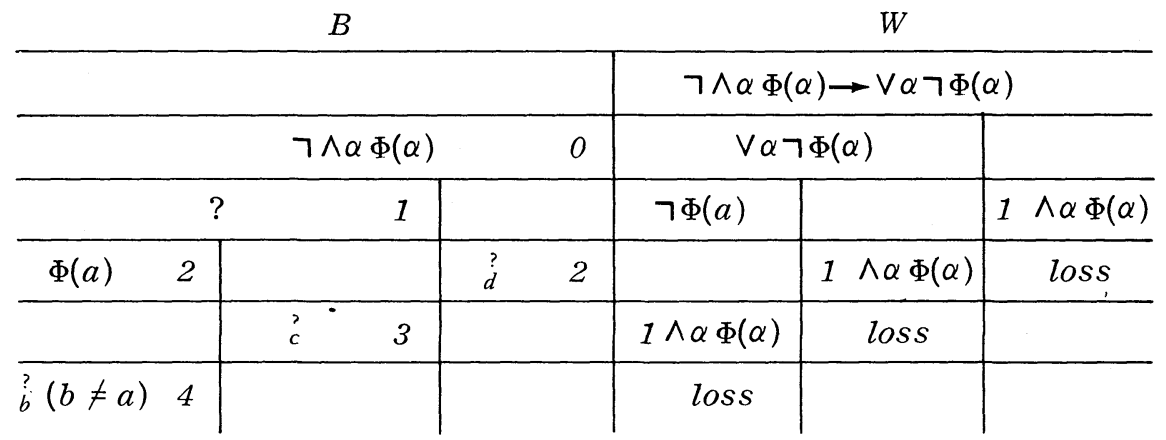

Here again $\Phi$ is supposed to be a prime formula. To each left (right) subcolumn of the $W$-column there corresponds a left (right) subcolumn of the $B$-column; the same holds for each subcolumn etc. The first subdivision arises from the fact that $W$ after the first attack of $B$ can choose between defending (by setting forth $\vee \alpha\urcorner \Phi(\alpha)$ ) or counter-attacking (by setting forth 
$\wedge \alpha \Phi(\alpha)$ )。 In the latter case he looses as soon as attacked by $B$ (he can not make good later for the omitted defence against $B$ 's first attack without violating the structural rule as there are rounds left open below the first round in which the defence-move would have to be made). In the former case where $W$ chooses defence after the first attack of $B$ the left subtableau again splits up as soon as $B$ has made his attack against $\vee \alpha\urcorner \Phi(\alpha)$ because now $W$ can either choose to defend against this attack (by taking an $a$ and setting forth $\neg \Phi(a)$ ) or to counter-attack $B$ 's formula $\neg \wedge \alpha \Phi(\alpha)$ etc. $W$ looses in all cases. As the tableau takes into account all possible moves of $W$ there can not exist a $W$-win-strategy and therefore the formula in question is not valid under the definition given. In the leftmost case $B$ has to make sure that in his last attack against $W^{\prime}$ s $\wedge \alpha \Phi(\alpha)$ he chooses an i.c. different from that one chosen by $W$ before (in his defence-move $\urcorner \Phi(a)$ ). This shows that "silly reactions" of $B$ may lead to a win of $W$ even if there exists no $W$-win-strategy. In the same way $W$ may loose a dialogue about a valid formula because he chooses a wrong strategy. This of course was to be expected: If the application of the game-rules would be mechanical we would have an effective decision precedure contradicting the recursive unsolvability of quantification theory.

The schema-interpretation of the tableau used to prove the non-validity of the formula in our last example requires an additional consideration: In none of the cases $B$ reaches a win-position after a finite number of steps because in principle $W$ can repeat (in all 3 cases) his attack against the formula $\urcorner \wedge \alpha \Phi(\alpha)$ of the $B$-column an arbitrary number of times. But $W$ can never win as long as $B$ answers again and again in the way described in the tableau. Therefore this is a situation where $B$ wins because he can prevent $W$ from winning in a finite number of steps. All the dialogues are formally of infinite length. The words "loss" inserted within the subcolumns of the $W$-column have to be interpreted in this sense.

If for the moment we take it for granted that the concept of validity defined coincides with intuitionistic validity then this example illustrates how the method of subtableaux (usually to be combined with an additional consideration of the kind just described) can be used to show the intuitionistic unprovability of certain formulas. This procedure is in most cases simpler than the other known methods.

In case the method is to be used to represent a fixed $B$-strategy (as in our last example) or a fixed $W$-strategy there is another item to be mentioned: Whenever that player all of whose moves have to be taken into account attacks a $\wedge$-formula or defends a $\vee$-formula he can choose the i.c. in infinitely many ways. Therefore strictly speaking we would get an infinite number of subtableaux (in our example in round 2 of the leftmost case). But it can easily be seen that this situation reduces to a finite number of possibilities consisting of 2 classes: the one class contains the choices of an i.c. already occuring in the tableau before that move; and the other class contains the choices of a new i.c. In the latter case only one arbitrary new i.c. has to be taken into consideration.

For the completeness proof no use will be made of the method of sub- 
tableaux. Rather the different dialogues emerging from a certain initial position will be represented as separate branches of a tree formally depicting a strategy chosen by one of the two players.

Remark 5.2. Special attention should be paid to the threefold asymmet$r y$ between $B$ and $W$ : the first is mirrored by the basic rule which introduces a difference in handling prime formulas; the second is given by the structural rule which allows only $W$ the repetition of attacks; the third follows from the difference in the definition of "win" and "loss" for $B$ and $W$.

Remark 5.3. The concepts of open and closed round as introduced by K. Lorenz and the precise formulation of the operations of opening and closing rounds is essential. It can be shown that the original presentation of the game-theory as given by $\mathrm{P}$. Lorenzen which does not make use of this concept leads to inadequate results. One could e.g. challenge Lorenzen's claim that his formulation gives the intuitionistic logic by the following counter-example ( $W$-win-strategy for double negation elimination): ${ }^{4}$

\begin{tabular}{c|c}
$B$ & $W$ \\
\hline$\urcorner p$ & $\neg\urcorner p \rightarrow p$ \\
$p$ & \urcorner $p$ \\
& $p$
\end{tabular}

Here attacks and counter-attacks as well as defences are inserted without distinction of rounds and use is made only of the fact that $W$ can first decide to counter-attack $\neg \neg p$ and later to defend his original formula by using the $p$ that has been set forth by $B$ in his last attack. Using the methods of rounds this case is to be represented in the following way:

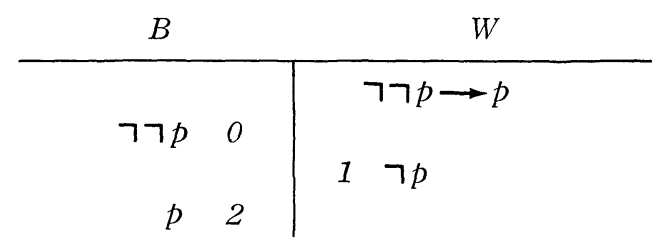

This time $W$ looses; the use of $p$ to close round 1 would violate part 3 ) of $\mathbf{S}$ because there are later open rounds 2 and 3 which can not be closed but which had to be closed before a closure of round 1 were allowed.

Remark 5.4. From the point of view of adequacy the question naturally arises whether an intuitive justification can be given for the rules of the (intuitionistic) logic-game. Here a distinction has to be made between the structural rule and the other rules.

As far as the structural rule is concerned the answer is negative. The best one can do e.g. with respect to part 2) of $S$ is to bring forward certain plausibility arguments: $W$ can "increase his knowledge" in the course of a dialogue because of new informations he gets by the prime formulas set

4. This simple negative example was brought to my attention by Mr. W. Essler, Munich. 
forth by $B$. As has already been shown in the first example it may happen that $W$ is successful in his second attack against a formula of $B$ because $B$ 's reaction to his first (unsuccessful) attack provides $W$ with the formula needed.

But of course considerations of this kind to not justify this rule as necessary. If it is dropped one gets other logical systems. Furthermore there is no intuitive justification at all for not allowing $W$ a repetition of defences as well. As has been shown by K. Lorenz the admission of defence-repetition produces the classical logic-game. Therefore there seems to be no possibility to mark out on the game-theoretical basis the intuitionistic logic as the "effective" or "real" logic against the classical one.

So it has to be admitted that the structural rule contains an element of arbitrariness. But it is exactly this element of arbitrariness which makes the game-theoretical semantics more flexible than the usual one so that it can be used to define validity-concepts for different svstems of logic.

With respect to the other rules an intuitive justification can be given though a somewhat more complex consideration seems to be needed than suggested by the remarks of P. Lorenzen. As far as $L$ is concerned one can agree that in case of $\wedge, \vee$ and $\vee$ the rules are in accordance with intuitionistic ideas proper: It has been suggested by intuitionists that for the meaningassignment to logical signs the concept of truth has to be replaced by the concept of proof. E.g. a $\wedge$-sentence is to be considered as proved if both $\wedge$-members are proved or a $\vee$-sentence is to be considered as proved if an object is "effectively given" and the sentence proved for this object. The parts 1),2) and 6) of the logical rule of the game can be considered as a translation of this idea into the language of game-theory (with additions to be made similar to those for the other cases below). In the case of the other 3 logical signs Lorenzen's game-theoretical interpretation (parts 3), 4) and 5) of L) gives a generalization of this approach. We take $\rightarrow$-formulas as an example to discuss the intuitive justification in these cases.

Suppose $W$ as global proponent has presented the initial formula $\Phi_{1} \rightarrow \Phi_{2} . \quad B$ can attack by setting forth $\Phi_{1}$. W has a choice between counter-attack and defence. Suppose he chooses the first and succeeds, i.e. he wins the "attack-dialogue" against $\Phi_{1}$. As $B$ is the representant of the satisfiability claim $W$ has thereby shown the unsatisfiability of $\Phi_{1}$. It is in sufficient accordance with the common usage of " $\rightarrow$ " to say in such a case that $\Phi_{1} \rightarrow \Phi_{2}$ has been established as valid: This part of the rule $L$ 4) amounts to the acceptance of the "ex falso quodlibet" for the definition of " $\rightarrow$ " (therefore it is not possible on this basis to introduce a validity concept for minimallogic, at least not without changing the logical rule). Suppose $W$ chooses the second alternative and wins the "defence-game" about the $\Phi_{2}$. He has thereby shown that no counterexample for $\Phi_{2}$ can be constructed if the satisfiability of $\Phi_{1}$ is taken for granted. This again is in accordance with the intended meaning.

The situation is different if $B$ is the global proponent who presents this formula in the first step. He claims only satisfiability and succeeds in "proving" this if he is able to show either the non-validity of $\Phi_{1}$ (by con- 
structing a counterexample against $W$ 's attack) or the satisfiability of $\Phi_{2}$ in case $\Phi_{1}$ is granted as valid. Again this is in accordance with the presystematic meaning. But it shows that a different kind of intuitive justification has to be given for the game-rule $L 4$ ) depending on whether $W$ or $B$ presents the $\rightarrow$-formula in the zero-step. The situation is even slightly more complicated because for $W$ (though not for $B$ ) it does make a difference whether the $\rightarrow$-formula is presented in the first step or later: if later then $W$ 's validity-claim is relative to certain "parameters" entering in this claim, namely the prime formulas that have been set forth before by $B$. Speaking in terms of sentences instead in terms of formulas we can put it this way: $W$ in this case does not claim validity outright but only validity on the assumption that certain elementary sentences are proved. The reasoning needed to justify the other parts of the logical rule are analogous.

The intuitive motivation for the basic rule is obvious: $W$ as representant of the validity-claim (as global proponent) or invalidity-claim (as global opponent) can not be allowed to base this claim on the assumption of special formulas (sentences) whereas $B$ in the dual position of a representant of the satisfiability - or rejectability-claim can do this.

For the definition of "win" and "loss" we have a straightforward justification too: The validity-claim can be accepted as established if this has been done in a finite number of steps.

So the only "intuitive gap" lies in the structural rule. It should not be overlooked that part 3) of this rule contains more than a precise description of the order in which the open rounds have to be closed and thereby another kind of an "intuitive gap": As a round opened by an attack against a 7 -formula can never be closed this part 3) bars all open rounds from ever being closed. The example $\neg \neg p \rightarrow p$ of remark 5.3 is an illustration of this point. This stipulation can hardly be justified in a different way than by pointing out that only by accepting it one can get a "reasonable" logic-game.

The artificiality in the structural rule is the price which has to be paid to get a more flexible concept of logical truth that is applicable to different logical systems and at the same time independent from certain problematic basic assumption mentioned in the beginning.

Stretching the idea of "how to determine the meanings of logical signs" somewhat more one could think of using the introduction-elimination-rules of a system of natural deduction as an alternative semantical approach as well. The artificiality in this case would consist in the well-known "structural restrictions" needed to prevent the rules from producing nonsense. What makes the game-theoretical approach more interesting are the connections it brings about between logic and another field of mathematical research and the fruitful applications to various classes of problems like the question of constructivity, intuitionistic unprovability and perhaps even consistency.

Remark 5.5. With respect to the definition of validity given the question arises whether the reference made within this definition to all possible moves of $B$ (consistent with the rules) introduces a non-constructive element. Using Brouwer's terminology one can say that we have here a uni- 
versal quantifier ranging over free choice sequences. A dialogue emerging from a given initial position on the basis of a fixed $W$-win-strategy consists of a sequence of arbitrary choices made by $B$ restricted by the "spread law" R. We can not enter here into a discussion of the basically philosophical question of constructivity. All we can say is this: if two things are admitted, namely (a) that a comparative concept of constructivity should be given the preference to a classificatory one, and (b) that the concept of a free choice sequence is more constructive than the concept of an arbitrary set then the game-theoretical concept of validity (and analogously of invalidity etc.) is more constructive than the corresponding concept of the Bolzano-Tarski-approach. ${ }^{5}$

Quite apart from this question the following proof may be called quasiconstructive because it shows how a given $W$-win-strategy ("given" either by a system of nested subtableaux or by a strategy-tree to be described later) can be transformed effectively in a derivation within a suitable calculus.

6. We now come to the formulation of a calculus of intuitionistic quantification theory. This calculus, called $S_{\text {int }}$, is apart from the symbolism and a minor detail identical with that given by K. Lorenz in [1], p. $102 \mathrm{ff}$. We use the following signs: $a, \mathscr{L}$. . are variables of the metalanguage designating arbitrary sequences of formulas. The symbol $\Gamma$ will be used to designate either the empty formula or expressions of the type $[\Phi]$ with a (non-empty) formula inside. Expressions of this latter kind will sometimes be called bracket-expressions or improper formulas. $\Phi\left(\begin{array}{c}\alpha \\ a\end{array}\right)$ designates the result of substituting the i.c. $a$ for the free occurrences of the variable $\alpha$ in $\Phi$. The arrow $\Rightarrow$ is used to communicate rules of derivation. The semicolon; is used to separate premisses in the rules. The signs of the object language consist of the usual logical apparatus plus the following additional symbols: *, "A" (always with two formulas as indices, e.g.: $A_{\Phi_{1}}, \Phi_{2}$ ), the comma "," and the two brackets "[" and "]". As before " $a$ " is always used to designate an i.c. (analogously " $b$ ", " $c$ " etc.) and " $p$ " stands for an arbitrary prime formula.

The formula expressions of the calculus are of the type $\alpha * \Phi$ or $\alpha * \Gamma$, called tableau-sequents. The first type could be called a $B$-tableau-sequent and the second a $W$-tableau-sequent. The star $*$ is called the sequent-symbol, the sequence of formulas preceding it in a sequent is called the antecedent and the (proper or improper) formula following it the succedent.

The axiom schema as well as the rules are to be applied irrespective of the order of the formulas in the antecedents (this stipulation can be rendered precise with the help of the concept of "cognate," comp. S. C. Kleene, IM, p. 480).

5. We shall come back to the more technical aspect of this question in the course of the completeness proof. 
Axiom schema: $\quad a, p * p$

Rules:

(1) (a) $a *[\Phi] ; a *[\Psi] \Rightarrow a * \Phi \wedge \Psi$

(b) $\quad a *\left[\mathbf{A}_{\Phi, \Psi}\right] \Rightarrow a * \Phi \vee \Psi$

(c) $a, \Phi *[\Psi] \Rightarrow \alpha * \Phi \rightarrow \Psi$

(d) $\quad a, \Phi * \Rightarrow a *\urcorner \Phi$

(2) (a) $\quad \alpha *\left[\Phi\left(\begin{array}{l}\alpha \\ a\end{array}\right)\right] \Rightarrow \alpha * \wedge \alpha \Phi$

(b) $\quad a *[\Phi] \Rightarrow a * \vee \alpha \Phi$

(3) (a) $a, \Phi \wedge \Psi, \Phi * \Gamma \Rightarrow a, \Phi \wedge \Psi * \Gamma$ $a, \Phi \wedge \Psi, \Psi * \Gamma \Rightarrow a, \Phi \wedge \Psi * \Gamma$

(b) $\quad a, \Phi \vee \Psi, \Phi * \Gamma ; a, \Phi \vee \Psi, \Psi * \Gamma \Rightarrow a, \Phi \vee \Psi * \Gamma$

(c) $\quad a, \Phi \rightarrow \Psi, \Psi * \Gamma ; a, \Phi \rightarrow \Psi * \Phi \Rightarrow \not{a}, \Phi \rightarrow \Psi * \Gamma$

(d) $a, \neg \Phi * \Phi \Rightarrow a, \neg \Phi * \Gamma$

(4) (a) $a, \wedge \alpha \Phi, \Phi\left(\begin{array}{l}\alpha \\ a\end{array}\right) * \Gamma \Rightarrow \alpha, \wedge \alpha \Phi * \Gamma$

(b) $\quad a, \vee \alpha \Phi, \Phi\left(\begin{array}{l}\alpha \\ a\end{array}\right) * \Gamma \Rightarrow a, \vee \alpha \Phi * \Gamma$

(5) (a) $\alpha * \Phi \Rightarrow \alpha *[\Phi]$

(b) $\quad a * \Phi \Rightarrow a *\left[\mathbf{A}_{\Phi, \Psi}\right]$

$$
a * \Psi \Rightarrow a *\left[\mathrm{~A}_{\Phi, \Psi}\right]
$$

(c) $\quad \alpha * \Phi\left(\begin{array}{l}\alpha \\ a\end{array}\right) \Rightarrow a *[\Phi]$

In the rules (2) (a) and (4) (b) the i.c. a must not occur in the sequent which is the conclusion of that rule.

It has been proved in [1] that (a calculus immediately seen to be equivalent with) $S_{\text {int }}$ is equivalent with Kleene's intuitionistic calculus $G 3$ (IM, p。 $480)$ in the sense that $* \Phi(\Phi *)$ is derivable in $S_{\text {int }}$ if $\rightarrow \Phi(\Phi \rightarrow)$ is derivable in G3 (" $\rightarrow$ " being the sequent-symbol of G3). As $G 3$ is equivalent in the well-known way with a formulation of intuitionistic quantification theory (e.g. with Heyting's or Kleene's) it is sufficient to prove the following:

Theorem 1. If $\Phi$ is valid in the sense of $D f_{1}$ then the expression $* \Phi$ is derivable in $S_{\mathrm{int}}$.

Proof: (I) As a preparatory step we choose a slightly different way of representing dialogues: Instead of inserting all (symbols for) moves into one and the same tableau we split the tableau up in a linear sequence of tableaux. The first tableau of this sequence contains the formula having been set forth in the zero-step only (in our case: it contains nothing besides the formula $\Phi$ in the right upper corner). Each of the other tableaux con- 
tains all the expressions occuring in its immediate predecessor plus the symbol for the next move. This sequence can be of finite or of infinite length. The elements of this sequence depict the momentary subsequent positions of the whole dialogue. Every sequence of this kind therefore represents a concrete dialogue.

We think of this sequence as written in a vertical order, starting at the bottom.

(II) Next we represent strategies with respect to a certain initial position by trees. Trees representing $W$-win-strategies will be transformed by successive steps into proof trees within $S_{\text {int }}{ }^{6}$

A strategy-tree (S.T.) associated with a certain initial position (and thereby with the formula occuring in this position) can be of one of the following 4 types: ${ }^{7}$

1) A Black-White-Strategy-Tree (BWS.T.): This is the degenerate case whe re both of $B$ and $W$ have chosen a fixed strategy. From these choices a concrete dialogue emerges. We can therefore identify a BWS.T. with a sequence described in (I).

2) An Open-Strategy-Tree (OS.T.): Here neither $B$ nor $W$ has chosen a fixed strategy. The tree has therefore to represent all possible moves and counter-moves. The origin consists of the tableau containing the initial formula (on the right or on the left corner). To each tableau $T$ we add as immediate upper tableau (i.u.t.) all those tableaux which are $R$-successors of $T$ (the positions of the dialogues hereby being identified with the tableaux as their formal representants). If there are several i.u.t. $T_{i}$ of $T$ we arrange them on the basis of a lexicographical ordering of the expressions representing the new move in $T_{i}$.

By a branch of this tree we understand a sequence of tableaux beginning with the origin and containing to each given tableau exactly one of the i.u.t. Each branch apparently represents one possible dialogue starting from the given initial position and each such dialogue is represented by one branch.

In general, an OS.T. will be infinite for two different reasons: It will contain some branches of infinite length (if to each $T$ on that branch an $R$ successor is defined and therefore the dialogue represented by it has no end position). It will be remembered that such a branch expresses a loss for $W$

6. I should like to emphasize that the following tree-construction was suggested to me by the two Lemmas 6 (p. 72ff.) and 9 (p. 99ff.) in [1]. On the other hand only this tree-construction seems to make the completeness - (and soundness-) property of $S_{\text {int }}$ explicit and at the same time simplifies the treatment by reducing what has to be proved to four critical cases dealt with in Lemma 1 to Lemma 4 below. The construction may therefore be considered as a oompletion and elaboration of the validity- and soundness-claim made in that thesis.

7. In the following section a more precise description of trees could be given. As it would not affect the proof we do without it. The reader who is interested in a precise characterization can easily obtain it from the material available in the literature. 
(rather than a draw). Furthermore it will contain infinite bifurcations whenever a $\wedge$-formula is attacked or a $\vee$-formula is defended because the i.c. used for this move can be chosen in infinitely many different ways.

3) A Black-Strategy-Tree (BS.T.) is a tree differing from that one mentioned in 2) in this respect: for all tableaux in $M_{B}$ the i.u.t. is uniquely determined by the strategy chosen.

4) A White-Strategy-Tree (WS.T.) is to be constructed similar to that in 3). Here the i.u.t. of a $T_{i} \in M_{W}$ is uniquely determined.

A BS.T. as well as a WS.T. can exhibit the same twofold infinity as an OS.T. with the difference that this time the infinite bifurcation can only arise when the counter-player (i.e. the player who has not chosen a fixed strategy) makes the attacks or defences mentioned.

If a branch of a tree is of finite length the uppermost tableau is called peak-tableau. It represents an end position favourable for one of the two players. Every tree is subdivided into levels; to each level a natural number is assigned as rank, the origin having the rank $O$ and the i.u.t. of a tableau of rank $n$ belonging to the level with rank $n+1$.

For the proof we suppose that the origin consists of a tableau containing $\Phi$ only in the right upper corner. If $W$ has a win-strategy for this initial position the corresponding WS.T. becomes a White-Win-Strategy-Tree (WW.T.). According to the definition of "win" for White this player wins every dialogue in finitely many steps no matter what moves are made by $B$.

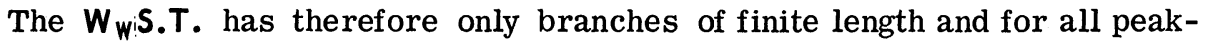
tableaux $T_{i}: T_{i} \in M_{B}$. We call this tree $\operatorname{Tr}(\Phi)$. The number of tableaux lying on the branch of greatest length is called the order of $W$ 's win-strategy represented by that tree. It is the smallest number $n$ that can be given at the beginning of the dialogue such that $W$ will win after ut most $n$ steps. This concept is not necessary for the following proof; it rather serves to get additional result.

Remark 6.1. The tree-representation may be used as a tool towards a clarification of the question of constructivity. Branches of a strategy-tree represent. free choice sequences. In case of an OS.T. the choices are made alternatingly by both players; in case of a BS.T. or WS.T. the choices are made by the counter-player. Suppose now the following numbering has been introduced: a gödelnumbering gn of formulas, an assignment of rank-numbers to the tree and an additional number-representation of the results of the choices made by the players so that the free choice sequences become number theoretic functions (this e.g. can be done with the help of an additional gödelization of the reduced tableaux introduced below which replace the original tableaux in the tree). We consider a statement of the form that a dialogue originating from an initial formula set forth by $W$ and developing on the basis of a fixed $W$-strategy $S$ and a set of free choices made by $B$ reaches an end position favourable for $W$ after $n$ steps. Under the numbering we get a 3-placed relation between a number theoretic choice sequence $\alpha$, a rank number $2 y+1$ and a gn $x$ of the formula in question. As it is effectively decidable whether a dialogue has at a certain moment reached an end position which means win for $W$ we get a recursive relation $R(\alpha, y, x)$ 
between a numbertheoretic function and two numbers. The statement that the strategy $S$ chosen by $W$ is a win-strategy for the formula in question means the same as: there exists to every choice sequence produced by the moves of $B$ (relative to the fixed $S$ ) a step-number $n$ such that after $n$ steps a win-position is reached for $W$. In terms of the numbering this becomes: $\wedge \alpha \vee y R(\alpha, y, x)$ with $R$ recursive. ${ }^{8}$ If the phrase "there exists a $W$-winstrategy ...' is interpreted in the non-constructive way then the statement that the formula with gödelnumber $x$ is valid becomes even more involved, namely: $\vee F \wedge \alpha \vee y R(F, \alpha, y, x)$ whereby $F$ represents the function called "strategy." The analogous representation of effective satisfiability would be more involved, namely: $\wedge \alpha(\vee y R(\alpha, y, x) \vee \wedge z N(\alpha, z, x)$. This is due to the fact that dialogues without end position are part of the $B$-win-strategy.

(III) We now replace all the tableaux on the $\mathrm{W}_{W}$ S.T. $\operatorname{Tr}(\Phi)$ by their socalled reductions (introduced in [1]). Roughly speaking a reduced tableau $\bar{T}$ is obtained from a tableau by eliminating from $T$ everything that is irrelevant for the further course of the dialogue and inserting in the open rounds symbols to designate the possible defence-moves in these rounds. More exactly $\bar{T}$ is obtained from $T$ by the following operations:

a) cancel all numbers indicating moves or rounds; b) cancel all challenges (a challenge can never be attacked); c) cancel all formulas of the $W$-column that have already been attacked (they can not be attacked any more); d) insert the following special symbols $d$ in the open rounds; $m$ be an arbitrary move, $m^{\prime}$ an attack against $m$ and $m^{\prime \prime}$ a possible defence of $m$ against $m^{\prime}$ ( $m^{\prime \prime}$ always occurs in the same round as $\left.m^{\prime}\right)$ : If $m^{\prime}$ is an attack against a $\wedge, \rightarrow-$ or $\wedge$ - formula then $m^{\prime \prime}$ is uniquely determined and $d$ shall be the expression $\left[m^{\prime \prime}\right]$. If $m^{\prime}$ is an attack against a 7 -formula then $d$ is the expression [ ]. If $m$ is a $\vee$-formula $\Phi \vee \Psi$ then $d$ is $\left[\mathbf{A}_{\Phi, \Psi}\right]$ ( "A" for "alternative"). If $m$ is the $\vee$-formula $\vee \alpha \Phi$ then $d$ is the same as [ $\Phi$ ] (so if the existential quantifier was not vacuous, the formula within the bracket contains the free variable $\alpha$ );

(We call [ ] the empty bracket expression. As in the case of the calculus we call every sequence of symbols beginning and ending with brackets an improper formula or a bracket expression. We use $\Gamma^{\#}$ in the same sense as $\Gamma$ but this time including the empty bracket (so $\Gamma^{\#}$ is either the empty formula or a bracket expression with a proper formula inside or the empty bracket).)

e) replace the horizontal line on top of the tableau by an arrow pointing to the left if $T \in M_{B}$ and to the right if $T \epsilon M_{W} ;$ f) arbitrary permutations of proper formulas and of proper formulas with bracket expressions are allowed as long as the order of bracket expressions among themselves is maintained (this holds for both columns).

8. Because of this fact the game-theoretical concept of validity can also be related to the theory of constructive ordinals (vide S. C. Kleene: On the forms of the predicates in the theory of constructive ordinals (second paper); American Journ. of Math., Vol. LXXVII, No. 3, especially Theorem I, p. 417. 
By requirement e) the domain of moves is made explicit. This is necessary in case the difference in the number of open rounds on the two columns (which difference is always $O$ or 1 !) is $O$ because in this case one can not find out by the structure of a reduced tableau in whose domain it lies if it is not explicitly indicated. The restriction in $\mathrm{f}$ ) with respect to the order of bracket expressions is necessary because this order symbolizes the order of open rounds (which is essential in view of the structural rule). To get a certain kind of uniqueness in the representation we decide always to "push" the bracket expressions to the end of the reduced tableau so that they follow all proper formulas.

The reduction of the tableau on p. 89, used to exemplify the $W$-winstrategy for a quantificational formula, looks like this:

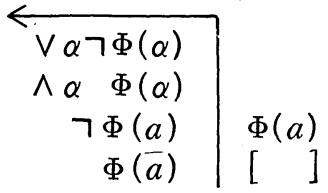

By the procedure described in (III) $\operatorname{Tr}(\Phi)$ has been transformed into another tree $\operatorname{Tr} *(\Phi)$ which consists instead of tableaux of their corresponding reductions. In the subsequent discussions we shall keep the original designations like "i.u.t." referring by them to the modified tree $T r *$.

We now extend the use of the concept of win-strategy: First we speak of a $W$-win-strategy for a tableau meaning, that $W$ in following that strategy will certainly $w$ in if the dialogue starts at a position represented by the given tableau. Secondly we even speak of a win-strategy for a reduced tableau. The latter way of talking is justified because if $W$ has a win-strategy for a tableau $T$ (whose reduction therefore is $\bar{T}$ ) then obviously this same strategy is a win-strategy for all the other tableaux with the same reduction $\bar{T}$ as well: these other tableaux differ from the given one only with respect to items that are irrelevant for the further course of the dialogue.

(IV) By a deduction string of a tableau-sequent $t$ in $S_{\text {int }}$ we understand a (finite or-infinite) sequence of tableau-sequents $t_{1}, t_{2}, \ldots$ such that $t$ is the first one and for each $i t_{i+1}$ is one of the premisses of $t_{i}$ for an application of one of the rules of $S_{\text {int }}$. If there is a last tableau-sequent in the string it is called the peak-tableau-sequent of that string. The number of tableausequents occurring in a string with finitely many tableaux is called the length of that string. By a deduction tree of a tableau-sequent $t$ in $S_{\text {int }}$ we understand an assemblage of deduction strings of $t$ which together form a deduction of $t$ within $S_{\text {int }}$. If all deduction strings are of finite length then we call the tree a finite deduction tree. The peak-tableaux of the strings which compose this tree are called the premises of that deduction tree. If all the premises of a finite deduction tree are axioms then the tree is called a proof tree of $t$. The length of the longest deduction string in a proof tree of $t$ is called the order of the $\left(S_{\text {int }}-\right)$ proof of $t$.

We shall show that the modified $W_{w}$ S.T. $T r *(\Phi)$ of the valid formula $\Phi$ can by successive steps be transformed into a proof tree of the tableausequent $* \Phi$ in $S_{\text {int }}$. We shall get the further result that the order of the 
proof of $* \Phi$ is not greater than the order of the win-strategy of $\Phi$ which was used in the construction of the $W_{W}$ S.T. of $\Phi$.

The tree $T r^{*}$ will be modified into a new tree whose reduced tableaux are of a very simple type: If the reduced tableau is in the domain of $B$ then in the new tree it will always be of this type:

(A)

$$
\leftarrow a \Psi
$$

If the reduced tableau is in the domain of $W$ then in the new tree it will always be of this type:

$$
a \longdiv { \Gamma ^ { \# } }
$$

Hereby $a$ is in both cases a (possibly empty) column consisting of proper formulas only (i.e. no bracket expression occurs in it), $\Psi$ in (A) is one single proper formula and $\Gamma^{\#}$ in (B) is to be interpreted in the way described above (p. 99). We call this new tree $T r^{s}$ or more exactly $T r^{s}(\Phi)$ (" $s$ " for "simplified"). It will turn out that it differs from a proof tree in $S_{\text {int }}$ only in sy mbolism (namely the vertical line and the arrow in the reduced tableaux has to be replaced by the sequent-symbol $*$ and the columns of formulas by rows containing the same formulas).

The modifications to be made on $T r^{*}$ start at the origin of the tree. Step by step the modifications are carried through to the higher levels of the tree. But we immediately consider the two general cases that a given reduced tableau $T$ either is an element of $M_{B}$ or an element of $M_{W}$.

Case 1: $T \in M_{B}$. Then this reduced tableau is of type (A) described above. This is certainly true for the initial position (with $a$ empty) because the origin of $\operatorname{Tr}$ consisted of the tableau $\frac{\Phi}{\Phi}$ wich has in $\operatorname{Tr}^{*}$ the reduction $\leftarrow$ Ф

For the other cases this assertion about the structure of the reduced tableaux is part of the proof.

So we can assume that $T$ is:

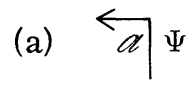

We distinguish two subcases.

Subcase 1.1: The principal $\operatorname{logical} \operatorname{sign}$ of $\Psi$ is one of the $5 \operatorname{signs} \wedge, \vee$, $\rightarrow, \neg, \vee .^{9}$ In these cases we leave the reduced immediate upper tableaux of (a) unchanged. So e.g. if $\Psi$ is the same as $\Psi_{1} \wedge \Psi_{2}$ then the next level of the tree contains the two reductions:

$$
\left(\mathrm{C}_{1}\right) \quad a \overleftrightarrow{\left[\Psi_{1}\right]} \quad a \overleftrightarrow{\left[\Psi_{2}\right]}
$$

To see that this is true one has to remember that on the next level the reductions of the tableaux depicting all possible moves of $B$ must appear; but

9. In the initial position $\Psi$ must have logical signs because $W$ can not use a prime formula in his first move. If $\Psi$ is a prime formula in a later position then (a) is the reduction of a peak tableau at which the process stops. 
$B$ in the situation described by (a) can only attack the $\wedge$-formula on the right by means of $\stackrel{?}{\ell}$ or $\stackrel{?}{r}$ so that we get the two reductions of $\left(C_{1}\right)$ (B can not choose to defend because there is no bracket expression on the left column representing a possible $B$-defence and he can not choose another attack because there is no other formula on the right column which he could attack besides the one mentioned in (a)).

Similarly if $\Psi$ is $\Psi_{1} \vee \Psi_{2}$ the i.u.t. of (a) is:

$$
\left(\mathrm{C}_{2}\right) \stackrel{a}{\left[\mathrm{~A}_{\left.\Psi_{1}, \Psi_{2}\right]}\right]}
$$

If $\Psi$ is $\Psi_{1} \rightarrow \Psi_{2}$ the i.u.t. of (a) is:

$$
\left(\mathrm{C}_{3}\right) \underset{\Psi_{1}}{a} \stackrel{a}{\left[\Psi_{2}\right]}
$$

If $\Psi$ is $\neg \Psi_{1}$ the i.u.t. of (a) is:

$$
\left(\mathrm{C}_{4}\right) \underset{\Psi_{1}}{a} \underset{[\mathrm{b}]}{\longrightarrow}
$$

If $\Psi$ is $V \alpha \Psi_{1}$ then the i.u.t. of (a) is:

$$
\left(C_{5}\right) a \overleftrightarrow{\left[\Psi_{1}\right]}
$$

(throwing a glance at the mapping of reduced tableaux to tableau-sequents below the reader can immediately see that we have got applications of the 4 rules (1) of $S_{\text {int }}$ and of (2)(b)).

Subcase 1.2: If $\Psi$ is $\wedge \alpha \Psi_{1}$ then the situation is different because the original tree $\overline{T r}$ as well as the modified tree $T r^{*}$ contain an infinite bifurcation at this node, as $B$ in his next move can attack the universal formula in infinitely many different ways. This bifurcation is removed with the help of the following:

Lemma 1. Suppose $W$ has chosen a fixed strategy $S$. Suppose further that in the dialogue to which this $S$ is applied a (position represented by a) tableau $T$ is reached whose reduction $T$ is:

$$
\leftarrow a \wedge \alpha \Sigma
$$

Then $S$ is a $W$-win-strategy for $T$ iff it is a $W$-win strategy for:

$$
\text { (ii) } \quad a \longdiv { [ \Sigma ( \begin{array} { l } 
{ \alpha } \\
{ a }
\end{array} ) ] }
$$

whereby $a$ is an i.c. not occurring in (i).

Proof: $S$ is a $W$-win-strategy for (i) iff it is one for all $R$-successors of it. As (ii) is one of these one has only to show that $S$ is a $W$-win-strategy for (i) if it is one for (ii). This simply follows from the fact that the infinitely many possible choices of individual constants in $B$ 's attack $\stackrel{\text { ? }}{a}$ against the formula $\wedge \alpha \Sigma$ can be represented by one single choice of "the worst kind for $W^{\prime}$ ', namely of one i.c. that has not yet occurred: If $B$ chooses another i.c. not occuring in (i) then we get a system of dialogues isomorphic to those starting with the position whose reduction is (i) (namely differing from these dialogues with respect to one i.c. only and therefore all ending 
with a win-position for $W$ as well). If $B$ ("is so silly to") choose(s) an i.c. which already occurs in (i) then the same holds. The only difference to the former case is this: it may happen that $W$ can win quicker than he could before (if the i.c. occurs within a prime formula of the $B$-column in (i) which can be used for a move leading to win for $W$ ). This at the same time shows that the implication of the Lemma from (ii) to (i) would not hold if the additional requirement in (ii) concerning the i.c. would be omitted.

Going back to our tree-construction we now decide to replace all the infinitely many reduced immediate upper tableaux of (a) by the reduced tableau:

$$
\left(\mathrm{C}_{6}\right) \quad \alpha \overleftrightarrow{\left[\Psi_{1}\left(\begin{array}{l}
\alpha \\
c
\end{array}\right)\right]}
$$

whereby $c$ is the alphabetically first i.c. not occuring in the given reduction (a).

Together with the elimination of all the other i.u.t. of (a) all subtrees having them as origins are eliminated as well. By Lemma 1 the new tree represents a win-strategy of $W$ iff the old tree represents such a win-strategy. As the tree has only been trimmed and nothing added the order of the win-strategy has not been increased by this manipulation.

(Under the mapping mentioned before we now got an application of the rule $(2)(\mathrm{a})$ of $\left.S_{\text {int }}\right)$.

Case 2: $T \in M_{W_{W}}$. Then the reduced tableau $T$ is of type (B). This holds for the situation after the first step as shown by the 6 cases $\left(C_{1}\right)$ to $\left(C_{6}\right)$. That it holds for the other cases as well is part of the proof. So we assume that $T$ which may occur on level with rank number $r$ is:

$$
\text { (b) } \quad a \overleftrightarrow{\Gamma^{\#}}
$$

(the $\Gamma^{\#}$ instead of $\Gamma$ is to be used because of the cases $\left(C_{4}\right)$ ).

This case is different in principle from case 1 in the following respect: A strategy chosen by $W$ is a win-strategy for a position in the domain of $B$ if it is a win-strategy for all $R$-successors of that position. This was the general situation in case 1 . On the other hand such a $W$-strategy is a winstrategy for a position in $W^{\prime}$ 's domain if it is a win-strategy for at least one $R$-successor of that position (because now it is up to $W$ to make the proper choice). This is the situation of case 2 .

If the position is like that described in (b) two classes of possibilities have to be taken into consideration: The given $W$-strategy can either prescribe defence for the next move (of course only if the symbol in the $W$ column of (b) expresses a possible defence that can be realized) or it can prescribe attack against one of the formulas occurring in the $W$-column of (b). As the tree represents a fixed strategy chosen by $W$ only one special case of these possibilities applies (i.e. the i.u.t. of the given one is uniquely determined).

Subcase 2.1: The $W$-strategy $S$ prescribes defence. Then the tree is left unchanged. So if the $\Gamma^{\star t}$ in (b) is of kind $[\Sigma]$ and $\Sigma$ does not contain a free variable then the i.u.t. in level with rank $r+1$ is: $\overleftarrow{d} \Sigma$. If $\Gamma^{\#}$ is $\left[\mathrm{A}_{\Sigma_{1}}, \Sigma_{2}\right]$ then the i.u.t. is either $\overleftarrow{a} \Sigma_{1}$ or $\overleftarrow{a} \Sigma_{2}$ (depending on what $S$ 
prescribes). If $\Gamma^{\#}$ is $[\Sigma]$ with a free variable $\alpha$ in $\Sigma$ then the i.u.t. is $\overleftarrow{a} \Sigma\left(\begin{array}{c}\alpha \\ a\end{array}\right)^{10}$ (if the previous $B$-attack was made against a $V$-formula with vacuous quantifier so that the quantification variable does not occur within the formula itself, then the first case applies here too). All the tableaux on level with rank $r+1$ are now of kind (A).

Subcase 2.2: The strategy chosen by $W$ prescribes attack against one of the formulas of $\alpha$. Again 4 subcases have to be distinguished.

Subcase 2.2.1: $S$ prescribes attack against a $\wedge-, v-$ or $\wedge$-formula. Then we eliminate the immediate upper tableaux on the level $r+1$ but leave the (reduced) tableaux on level $r+2$ unchanged. If e.g. $S$ prescribes an attack against a formula $\Sigma_{1} \wedge \Sigma_{2}$ then the i.u.t. of (b) is

$$
\text { either } \left.\left.(x) \begin{array}{c}
a^{\prime} \\
\Sigma_{1} \wedge \Sigma_{2} \\
{\left[\Sigma_{1}\right]}
\end{array}\right] \Gamma^{\#} \quad \text { or }(y) \begin{array}{c}
\Sigma_{1} \wedge \Sigma_{2} \\
{\left[\Sigma_{2}\right]}
\end{array}\right] \Gamma^{\#}
$$

being the column of formulas of $a$ different from $\Sigma_{1} \wedge \Sigma_{2}$ (and empty after the first move). In this situation the only move $B$ can make is to realize the possible defence designated by the bracket-expression on the left column. Therefore there is respectively only one i.u.t. of $(x)$ or $(y)$, namely

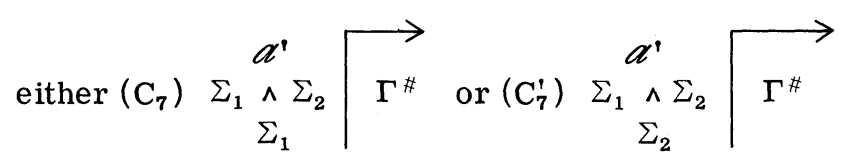

$S$ is a $W$-win-strategy for the former tableaux iff it is one for the latter. Therefore the elimination of the reduced tableau $(x)$ or $(y)$ of level $r+1$ does not do any harm; it just removes an unnecessary intermediate member from the modified $W$-win-strategy-tree.

If $S$ prescribes an attack against a formula $\Sigma_{1} \vee \Sigma_{2}$ then the i.u.t. of (b) is

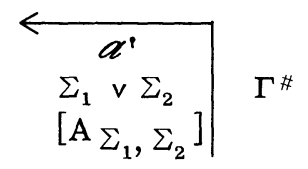

which is now being eliminated so that only its two i.u.t. on level $r+2$ are retained, namely

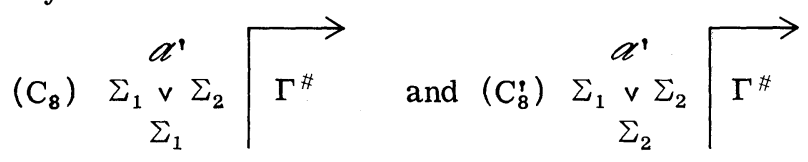

The justification is the same as before.

If finally $S$ prescribes an attack against a formula $\wedge \alpha \Sigma$ then we get by the same elimination procedure as immediate upper tableau of $(b)$ on level $r+2$

10. The i.c. $a$ is determined by $S$. 


$$
\begin{array}{ll} 
& \begin{array}{c}
a^{\prime} \\
\left(\mathrm{C}_{9}\right) \\
\wedge \alpha \Sigma \\
\Sigma\left(\begin{array}{l}
\alpha \\
a
\end{array}\right)
\end{array}
\end{array}
$$

whereby the i.c. $a$ is determined by $W$ 's attack.

Nothing has been changed with respect to the given win-strategy; but some branches of the strategy-tree have become shorter.

(Under the final mapping applications of the rules (3)(a),(b) and (4) (a) of $S_{\text {int }}$ have been obtained).

Subcase 2.2.2: $S$ prescribes to attack a formula $\vee \alpha \Sigma$ of the column $a$ in (b). On this node the tree again exhibits an infinite befurcation because on level $r+2$ there appear the reductions of the tableaux representing the defence moves of $B$ for all possible choices of individual constants. The following modifications are now made on the tree: First, the i.u.t. of (b) on level $r+1$, namely:

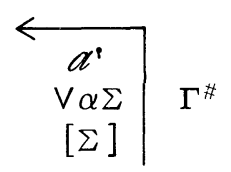

is eliminated. Secondly all the i.u.t. of this one on level $r+2$ are eliminated except:

$$
\begin{array}{lc}
\alpha^{\prime} & \longrightarrow \\
\left(\mathrm{C}_{10}\right) & \vee \alpha \Sigma \\
& \Sigma\left(\frac{\alpha}{c}\right)
\end{array} \Gamma^{\#}
$$

whereby $c$ is the alphabetically first i.c. not occurring in the given reduction (b). Thirdly of course all the subtrees having the eliminated reduced tableaux of level $r+2$ as origins are abolished too.

The elimination on level $r+1$ is justified as before. The justification for the second and third elimination step is based on the following:

Lemma 2. Suppose in a dialogue a position is reached whose corresponding reduction is:

$$
\text { (i) } a \longdiv { \Gamma ^ { \# } }
$$

Suppose further that $W$ has chosen a strategy $S$ which prescribes attack against a formula of the $B$-column $a$ which is of the kind $\vee \alpha \Sigma$. Then $S$ is a $W$-win-strategy for (i) iff it is a $W$-win-strategy for

$$
\text { (ii) } \begin{gathered}
a \\
\Sigma\left(\begin{array}{l}
\alpha \\
a
\end{array}\right)
\end{gathered} \longrightarrow \Gamma^{\#}
$$

whereby $a$ is an i.c. not occurring in (i).

Proof: By the assumption made on $S$ this strategy is a $W$-win-strategy for (i) iff it is one for the uniquely determined successor of (i), namely

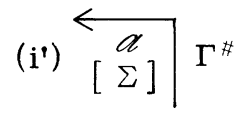


It now has only to be observed that $B$ can in this situation make no other kind of move than to defend. The proof from this point on is the same as in Lemma 1: all possible defence-moves of $B$ can be represented by a single one which is "of the worst kind" for $W$.

Because of this Lemma the treetrimmed by the procedures of this subcase still represents a full $W$-win-strategy for the given position. As in the former 3 cases the present modification only shortens certain branches of the tree without elongating others.

(Under the final mapping this case becomes an application of rule (4)(b)).

Remark 6.2. The procedures leading to $\left(\mathrm{C}_{6}\right)$ and $\left(\mathrm{C}_{10}\right)$ have the effect of removing the two kinds of infinite bifurcations from the tree. The elimination of the reduced tableaux on level $r+1$ in the last four cases prevent that the reduced tableaux have a more complicated structure than those of type (A) and (B).

Subcase 2.2.3: $S$ prescribes an attack against a formula $\Sigma_{1} \rightarrow \Sigma_{2}$ of the column $a$ in (b). The i.u.t. on level $r+1$ therefore is

$$
\begin{array}{c|c}
\multicolumn{1}{c|}{a^{\prime}} \\
\Sigma_{1} \rightarrow \Sigma_{2} \\
{\left[\Sigma_{2}\right]}
\end{array} \Sigma_{1}
$$

This again is a reduced tableau of an unwanted type having proper and improper formulas on both sides. This time a proper replacement is made on the tree (and not only a trimming as before) based on the following:

Lemma 3. Suppose in a dialogue a position is reached whose reduction is:

$$
\text { (i) } \quad \alpha \overleftrightarrow{\Gamma^{\#}}
$$

Suppose further $W$ has chosen a strategy $S$ which prescribes attack against a formula $\Sigma_{1} \rightarrow \Sigma_{2}$ occurring within $a$ on the $B$-column. Then $S$ is a $W-$ win-strategy for (i) iff it is a $W$-win-strategy for

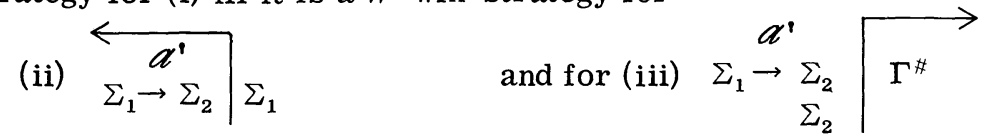

The orders which $S$ has as a win-strategy of (ii) and (iii) are each smaller than the order of $S$ as a win-strategy for (i).

Proof: By presupposition $S$ is a $W$-win-strategy for (i) iff it is one for the $R$-successor of (i) (uniquely determined by $S$ ), i.e. for

$$
\begin{array}{c|c}
\boldsymbol{a}_{1} \rightarrow \Sigma_{2} & \Sigma_{1} \\
\left.\Sigma_{2}\right] & \Gamma^{\#}
\end{array}
$$

It is therefore sufficient to relate ( $\left.i^{\prime}\right)$ with (ii) and (iii). In the situation described by (i') $B$ has the choice between a counter-attack against the formula $\Sigma_{1}$ or a defence by setting forth $\Sigma_{2}$. It is stated in this Lemma that these two cases can be treated separately so that $W$ 's strategy is a win-strategy for the original case iff it is one for each of these two cases. 
1) Suppose $S$ is a $W$-win-strategy for (ii) and (iii). Then $S$ is a $W$-winstrategy for (i') as well. For if $B$ in case (i') should decide to attack $\Sigma_{1}$ then $W^{\prime}$ 's strategy is the same as in case (ii) so that $B$ can not prevent $W$ from winning (by the assumption made on (ii)) as long as $B$ continues this "counter-attack dialogue" about the formula $\Sigma_{1}$. The additional improper formula $\Gamma^{\#}$ on the right in (i') can not be attacked by $B$ and therefore does not increase his chances of winning. If $B$ decides in case (i') to defend by setting forth $\Sigma_{1}$ again he can not prevent $W$ from winning (by the assumption made on (iii)) as long as he continues this "defence-dialogue." Now in general $B$ will make use of both possibilities (counter-attacking and defending). But still he can not prevent $W$ from winning as long as $W$ reacts in the one situation as determined by $S$ for (ii) and in the other situation as determined by $S$ for (iii). $B$ can not benefit by combining both possibilities because for $B$ there does not exist an "increase of information" obtained in the course of the dialogue (for this reason it would not be possible to "split up" a dialogue about a $\rightarrow$-formula asserted by $W$ in the way described in Lemma 3, because $W$ could win the complex dialogue consisting of two part-dialogues each of which he would loose separately).

2) Suppose $S$ is a $W$-win-strategy for (i'). Then it certainly is one for (iii) as well. $S$ being a $W$-win-strategy for (i') must be one for all $R$-successors of ( $\left.i^{\prime}\right)$, especially for that one in which $B$ has decided to defend in the next move by setting forth $\Sigma_{2}$. The reduction of this position differs from (iii) only by containing the additional formula $\Sigma_{1}$ on the right column. But this formula gives $B$ an additional possibility of counter-attacking. So if $W$ has a win-strategy in this case then a fortiori he has one for (iii).

Furthermore $S$ under this assumption is a $W$-win-strategy for (ii) as well. This time the fact has to be used that $S$ is a $W$-win-strategy for the successor of ( $\left.i^{\prime}\right)$ characterized by an attack of $B$ against $\Sigma_{1}$. The additional possibility (if it is one) for $W$ described by $\Gamma^{\#}$ in ( $i^{\prime}$ ) is only an apparent one that can not be used by $W$ as long as $B$ continues his attack-dialogue against $\Sigma_{1}$ : By setting forth $\Sigma_{1} W$ has opened a new round which must first be closed before $W$ can make use of the possible defence $\Gamma^{\#}$ which is already mentioned in (i) and therefore must go back to an earlier open round. So the fact that $W$ has a win-strategy for ( $\left.i^{\top}\right)$ can in this case not be based on $\Gamma^{\#}$ (here for the first time within the completeness-proof the regulation about closing open rounds comes into play).

As in a situation described by (i') $B$ can always try out both possibilities open to him (whereas in each of the cases (ii) and (iii) he has only one possibility), the order of $S$ as a $W$-win-strategy for (i') is certainly greater than the order of $S$ as a $W$-win-strategy of (ii) and as a $W$-win-strategy of (iii).

Going back to Subcase 2.2.3 we now replace the reduced tableau $(x)$ and the subtree originating in it by the two reduced tableaux

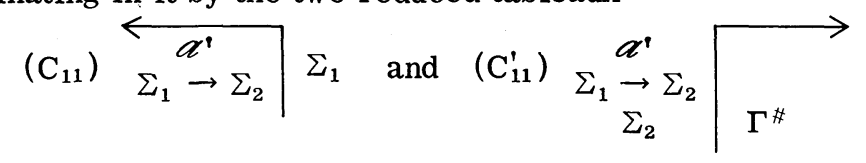

and the two (shorter) trees representing $W$-win-strategies for them. That this can always be done has been shown in Lemma 3. Special attention should be paid to the fact that $\left(C_{11}\right)$ contains a proper formula on the right (therefore the arrow goes to the left) and $\left(C_{11}^{\prime}\right)$ contains an improper formula on the right (so that the arrow goes to the right too). 
(Under the final mapping this has become an application of rule (3) (c) of $\left.S_{\text {int }}\right)$.

Subcase 2.2.4: $S$ prescribes a $W$-attack against a formula $\neg \Sigma$ of the $B$-column in (b). The i.u.t. of (b) on level $r+1$ is

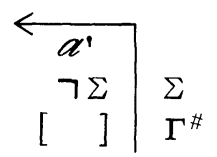

The simplification of the tableau is this time based on the following:

Lemma 4. Suppose in a given dialogue a position is reached whose reduction is

$$
\text { (i) } \quad a \overleftrightarrow{\Gamma^{\#}}
$$

Suppose further $W$ has chosen a strategy $S$ which prescribes attack against a formula $\neg \Sigma$ on the $B$-column. Then $S$ is a $W$-win-strategy for (i) iff it is a $W$-win-strategy for

$$
\text { (ii) } \leftarrow \begin{gathered}
a^{\prime} \\
\neg \Sigma
\end{gathered}
$$

Proof: The $R$ - successor of (i) is under the assumption made

$$
\text { (i') } \begin{array}{c|c}
a^{\prime} \\
\neg \Sigma \\
{\left[\begin{array}{l}
{[} \\
{[}
\end{array}\right]}
\end{array}
$$

So it is sufficient to relate ( $\left.\mathrm{i}^{\prime}\right)$ and (ii) by an iff-sentence. If $S$ is a $W$-winstrategy for (ii) then it is one for ( $\left.i^{\prime}\right)$ too because the empty bracket represents no additional possibility for $B$ whereas the $\Gamma^{\#}$ on the right in (i') may perhaps represent an additional defence-possibility for $W$ (but as it will turn out immediately this additional possibility is a spurious one).

If $S$ is a $W$-win-strategy for (i') then it is one for (ii) as well: First the omission of $\Gamma^{\#}$ in (ii) does not hurt. By attacking a $\neg$-formula $W$ has opened a round which can never be closed. On the other hand $\Gamma^{\#}$ already occurs in (i) and therefore can represent a possible defence (if at all) coming from an earlier open round only. Therefore $W^{\prime}$ 's win-strategy for (i') must be independent of it so that it need not be mentioned at all. Second the omission of the empty bracket in (ii) does not make a difference either because it represents no actual possibility of $B$ (but it would make a difference if on the $B$ column there were symbols for possible defences coming from earlier open rounds whose realization is barred by [ ], but admitted after removal of this barrier; however besides [ ] there appear proper formulas on the $B$ column in ( $\left.i^{\prime}\right)$ only). This again shows the importance of part 3 ) of the structural rule.

With the help of Lemma 4 the reduced tableau (y) is replaced by

$$
\left.\left(\mathrm{C}_{12}\right)^{\stackrel{a^{\prime}}{\neg \Sigma}}\right|_{\Sigma}
$$

and the tree originating in (y) by the tree which represents $W$ 's win-strategy for $\left(C_{12}\right)$. Actually the only modification to be made with respect to the other members of the tree consists in eliminating the same 2 items of $(y)$ 
that have been cancelled from $\left(C_{12}\right)$. Especially the order of the win-strategy has not been increased.

All the reduced tableaux obtained from applying the modifications in subcase 2.2 are either of type (A) or of type (B). So in every case we can apply the same procedures described in case 1 and case 2 again and again. After a finite number of steps the construction must come to an end, because by presupposition all the branches of the tree $\operatorname{Tr}(\Phi)$ and therefore of $\operatorname{Tr} *(\Phi)$ are of finite length and the modifications to be made lead to branches of the same or a smaller length. Apart from the symbols used the resulting tree $\operatorname{Tr}^{s}(\Phi)$ has become a proof tree of $S_{\text {int }}$. To get such a tree in the symbolism of this calculus one has only to perform the following mappings:

1) replace all $B$-tableaux $九 \Psi$ by $a * \Psi$

2) replace all $W$-tableaux a)
a) $a \overrightarrow{[\Psi]}$ by $a *[\Psi]$
b) $a \overleftrightarrow{\left[\mathrm{A}_{\Psi_{1}, \Psi_{2}}\right]}$ by $a *\left[\mathrm{~A}_{\Psi_{1}, \Psi_{2}}\right]$
c) $a \overrightarrow{[]}$ by $a *$

Hereby the $\alpha$ in the tableau-sequent is an (arbitrary) linear arrangement of the same formulas occurring within the column of the reduced tableau which is called $a$ too.

The cases described in $\left(\mathrm{C}_{1}\right)-\left(\mathrm{C}_{6}\right)$ have become applications of the rules (1) and (2); the situations dealt with in subcase 2.1 have become applications of the rules (5); and the cases described in $\left(C_{7}\right)-\left(C_{12}\right)$ have become applications of the rules (3) and (4). The reduced peak tableaux-representing win-positions for $W$-are in the domain of $B$ and therefore of type (A). The formula on the right must be a prime formula which must occur somewhere in the column of the left. This shows that the reduced peak-tableaux under the final mapping have become tableau-sequents falling under the axiom schema of $S_{\text {int }}$.

We have got the additional

Corollary. Suppose $\Phi$ is valid and the $W$-win-strategy for $\Phi$ is given (by means of a $\mathbf{W}_{W}$ S.T. or a system of nested subtableaux each of which is closed) then a proof of $* \Phi$ can effectively be constructed such that the order of this proof is not greater than the order of the given win-strategy.

Invalidity and soundness. $S_{\mathrm{int}}$ at the same time provides a proof-procedure for invalidity: If $\Phi$ is invalid then the tableau-sequent $\Phi *$ is provable in $S_{\text {int }}$.

The proof follows immediately from that of theorem 1 and the definition of invalidity (the latter meaning $W$-win-strategy for an initial position of

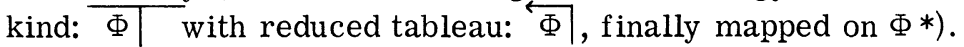

Theorem 2. (Soundness of $S_{\mathrm{int}}$ ) If $* \Phi(\Phi *)$ is provable in $S_{\mathrm{int}}$ then $\Phi$ is valid (invalid).

The proof is obvious: Tableau-sequents with a proper formula behind the $*$ are mapped on reduced $B$-tableaux (with arrow to the left) and tableausequents with an improper formula behind the $*$ are mapped on reduced $W$ - 
tableaux. In each application of rule $(1)(d)$ the empty space behind the $*$ has to be filled in with [ ]. The rules of $S_{\text {int }}$ are interpreted in this sense: "if there is a $W$-win-strategy for a tableaux whose reductions stand before the $\Rightarrow$ then the same holds for the tableaux behind the $\Rightarrow$ ". This apparently holds in all cases and especially-because of the iff-character of the four Lemmas-for the rules $(2)(a),(3)(c)$ and (d), (4)(b). Finally axioms are mapped on reductions of tableaux representing end-positions with win for $W$. From these facts the theorem follows.

7. Classical logic. As has been shown by K. Lorenz other logical systems are obtained by changing that part of the rule which we called "structural rule," leaving the logical rule and the basic rule unchanged. The classical logic game is obtained by permitting $W$ (and only him) besides the repetition of attacks the repetition of defences as well. As in the intuitionistic logic-game defence moves can be made only in order to close an open round, this change in the structural rule requires the introduction of a new type of rounds which are opened by defence moves (therefore called defence rounds by $\mathrm{K}$. Lorenz in distinction to the "attack-rounds") and always remain open for $B$ in the further course of the dialogue. No additional requirement for such openings of new rounds are made (analogous to those for closing rounds by defences). $W$ is allowed to open the same kind of defence rounds an arbitrary number of time.

With this modified rule of the game the concept of validity can be defined in literally the same way as before (the same holds for the other "semantical" concepts). It turns out that this concept coincides with classical validity. This can be shown by proving the soundness and completeness of a calculus $S_{\mathrm{cl}}$ formally equivalent to the classical version of $G 3$. It is somewhat surprising that the completeness proof given in 6 . can be carried over literally to the classical case. As we do not presuppose that the reader is familiar with the thesis of $\mathrm{K}$. Lorenz some indications will be made how to get $S_{\mathrm{cl}}$ from $S_{\text {int }}$. The reader can then carry out the proof without difficulty.

The only change that has to be made in $S_{\text {int }}$ is to insert in some of the rules names for arbitrary sequences of bracket-expressions. With $\mathcal{L}$ as a symbol designating such sequences (possibly empty) we make the following changes: in the axiom schema insert $\mathcal{L}$ between the $*$ and the $p$ of the succedent; in rules (1), (2) and (5) add an $\mathcal{\alpha}$ immediately behind the $*$; in rules (3) and (4) replace the $\Gamma$ by $\mathcal{L}$ (and in the premise of rule (3)(d) insert $\mathcal{L}$ immediately behind the $*$ ).

Reduced tableaux are introduced in an analogous way as before; the only change that has to be made is this: in the $B$-column only those possible defences have to be introduced which come from open rounds following the last defence round. No such restriction holds for the $W$-column (for $W$ a defence round is not an open round).

The reduced tableaux used in the completeness- and soundness proof will differ from the reductions of type (A) and (B) above (p. 25) by containing both additional bracket expressions on the right column. This is due to the fact that the strategy chosen by $W$ may tell him, for certain positions in his 
domain, to open a new defence round instead of making use of a possible defence coming from an earlier attack-round (thereby leaving possible defences in earlier attack-rounds in the stage of mere possibilities). As in the intuitionistic case the tree has to be left unchanged whenever the strategy chosen by $W$ prescribes defence (no matter what type of defence).

8. Non-intuitionistic subsystems of classical logic. As shown in [1] other logical systems are obtained by modifying the structural rule in other respects. One gets the anti-intuitionistic logic if one accepts the principle of defence repetition for $W$ (like in classical logic) but forbids attack repetitions for $W$. If complete symmetry is introduced between $B$ and $W$ as far as the structural rule goes (by allowing both of them to make one attack and one defence only) then the strict logic is obtained, which is a sublogic of the intuitionistic as well as of the anti-intuitionistic logic. Systems similar to $S_{\text {int }}$ and $S_{\mathrm{cl}}$-formally equivalent with gentzen-like calculi of sequents in the strict sense-can be constructed in an obvious manner: the rules for the strict $\operatorname{logic}$ calculus $S_{\mathrm{st}}$ are the same as that for $S_{\text {int }}$ except for not containing the formula with the logical sign in the premises of rules (3) and (4). And the rules of the anti-intuitionistic calculus $S_{\mathrm{ai}}$ are obtained from those of $S_{\mathrm{cl}}$ by exactly the same change of the rules (3) and (4).

The completeness- and soundness proof by means of tree-construction carries over to $S_{\mathrm{st}}$ and $S_{\mathrm{ai}}$. The only modification to be made concerns the analogon to Lemma 3 (p. 31 f.). Using the symbolism of that Lemma we get the following change (whereby for $S_{\mathrm{ai}}$ in addition the one bracket expression in (i), (iii) and (i') has to be replaced by a column of such expressions and in (ii) the same column to be inserted on the right side below the proper formula $\left.\Sigma_{1}\right)$ :

In ( $\mathrm{i}^{ }$), (ii) and (iii) the $\rightarrow$-formula has to be cancelled from the left (it can not be attacked a second time). Furthermore the column $\boldsymbol{\alpha}^{\prime}$ of formulas occurring in ( $\left.i^{\prime}\right)$ has to be subdivided exhaustively into two mutually exclusive subclasses $\alpha_{1}^{i}$ and $\alpha_{2}^{\prime}$ such that one of them ( $\alpha_{1}^{\prime}$ say) occurs on the left in (ii) and the other on the left in (iii). What remains to be proved is this: If $W^{\prime}$ 's strategy is a win-strategy for the (modified) reduced tableau (i') then it is one for the two reduced tableaux (ii) and (iii)-both modified in the two respects mentioned-as well. Suppose $B$ decides in case (i') only to attack $\Sigma_{1}$. Then by assumption $W$ has a win-strategy for this case. In order to succeed $W$ will normally need some attacks against proper formulas of the $B$-column $\alpha^{\prime}$. We call them $\alpha_{1}^{\prime}$ and take them as the $B$-column for (ii). But $W$ must by assumption be able to win in case (i') even if $B$ later decides to set forth $\Sigma_{2}$ in a defence move. For this case $W$ will again in the normal case need some attacks against formulas of the $B$-column. But they must all be different from those formulas of the $B$-column that have been attacked in order to win the part-dialogue beginning with $B$ 's attack against $\Sigma_{1}$ (i.e. they must be different from $\alpha_{1}^{\prime}$ ). This justifies the subdivision of formulas in two exclusive classes within (ii) and (iii). If there are formulas on the left column in (i') which $W$ does not need to attack in order to win with certainty, then these formulas can arbitrarily be assigned to the one or to the other subclass, e.g. to the second calling it $\alpha_{2}^{\prime}$ and using it in (iii) on the left. 


\section{REFERENCES}

[1] K. Lorenz, Avithmetik und Logik als Spiele. Doctorial thesis, Kiel 1961.

[2] P. Lorenzen, Ein dialogisches Konstruktivitätskriterium; in: Infinistic Methods, Proc. of the Symp. on Found. of Math., Warsaw 1959.

[3] P. Lorenzen, Metamathematik, Mannheim 1962.

Universität München

München, Germany 